\title{
SPI: The spectrometer aboard INTEGRAL
}

\author{
G. Vedrenne ${ }^{1}$, J.-P. Roques ${ }^{1}$, V. Schönfelder ${ }^{2}$, P. Mandrou ${ }^{1}$, G. G. Lichti ${ }^{2}$, A. von Kienlin ${ }^{2}$, B. Cordier ${ }^{3}$, S. Schanne ${ }^{3}$,
} J. Knödlseder ${ }^{1}$, G. Skinner ${ }^{1}$, P. Jean ${ }^{1}$, F. Sanchez ${ }^{5}$, P. Caraveo ${ }^{4}$, B. Teegarden ${ }^{9}$, P. von Ballmoos ${ }^{1}$, L. Bouchet ${ }^{1}$, P. Paul ${ }^{1}$, J. Matteson ${ }^{7}$, S. Boggs ${ }^{8}$, C. Wunderer ${ }^{2}$, P. Leleux ${ }^{6}$, G. Weidenspointner ${ }^{1,9}$, Ph. Durouchoux $^{3}$, R. Diehl $^{2}$, A. Strong ${ }^{2}$, M. Cassé ${ }^{3}$, M. A. Clair ${ }^{10}$, and Y. André ${ }^{10}$

1 Centre d'Étude Spatiale des Rayonnements, CNRS/UPS, BP 4346, 31028 Toulouse Cedex 4, France

2 Max-Planck-Institut für Extraterrestrische Physik, Postfach 1603, 85740 Garching, Germany

3 DSM/DAPNIA/SAp, CEA-Saclay, 91191 Gif-sur-Yvette, France

4 IASF, via Bassini 15, 20133 Milano, Italy

5 IFIC, University of Valencia, 50 avenida Dr. Moliner, 46100 Burjassot, Spain

6 Insitut de Physique Nucléaire, Université catholique de Louvain, 1348 Louvain-la-Neuve, Belgium

7 UCSD/CASS, 9500 Gilman Drive, La Jolla, CA 92093-0111, USA

8 Space Science Laboratory, University of California, Berkeley, CA 94720, USA

${ }^{9}$ Laboratory for High Energy Astrophysics, NASA/Goddard Space Flight Center, Greenbelt, MD 20771, USA

${ }^{10}$ CNES/CST, 18 avenue Édouard Belin, 31401 Toulouse Cedex 4, France

Received 19 July 2003 / Accepted 20 September 2003

\begin{abstract}
SPI is a high spectral resolution gamma-ray telescope on board the ESA mission INTEGRAL (International Gamma Ray Astrophysics Laboratory). It consists of an array of 19 closely packed germanium detectors surrounded by an active anticoincidence shield of BGO. The imaging capabilities of the instrument are obtained with a tungsten coded aperture mask located $1.7 \mathrm{~m}$ from the Ge array. The fully coded field-of-view is $16^{\circ}$, the partially coded field of view amounts to $31^{\circ}$, and the angular resolution is $2.5^{\circ}$. The energy range extends from $20 \mathrm{keV}$ to $8 \mathrm{MeV}$ with a typical energy resolution of $2.5 \mathrm{keV}$ at $1.3 \mathrm{MeV}$. Here we present the general concept of the instrument followed by a brief description of each of the main subsystems. INTEGRAL was successfully launched in October 2002 and SPI is functioning extremely well.
\end{abstract}

Key words. instrumentation: detectors, spectrographs - techniques: spectroscopic

\section{Introduction}

In 1993 the European Space Agency (ESA) selected a new scientific mission, INTEGRAL (International Gamma Ray Astrophysics Laboratory), devoted to gamma ray astronomy in the $3 \mathrm{keV}$ to $10 \mathrm{MeV}$ energy domain. Aimed at succeeding NASA's Compton Gamma Ray Observatory and the Russian/French Granat/SIGMA mission, the scientific objectives of INTEGRAL were centred on:

- stellar nucleosynthesis via detection, cartography and fine spectroscopy of sources (nearby supernovae, SN remnants or novae) and structures (galactic disk, galactic centre region) emitting radioactive gamma-ray lines such as those of ${ }^{56} \mathrm{Co},{ }^{44} \mathrm{Ti},{ }^{22} \mathrm{Na},{ }^{26} \mathrm{Al},{ }^{60} \mathrm{Fe}$ and the $511 \mathrm{keV}$ positron annihilation line;

- physics of compact objects such as pulsars, neutron stars or black holes in binary systems and massive black holes in the centres of active galactic nuclei;

Send offprint requests to: J. Knödlseder, e-mail: knodlseder@cesr.fr
- gamma-ray burst studies using fast detection and precise localisation at the arcmin level to trigger afterglow studies.

To reach these objectives, two main instruments were selected:

- the INTEGRAL imager (IBIS) with an angular resolution of $12^{\prime}$, allowing for source localisation with arcmin precision within a field of view of $9^{\circ} \times 9^{\circ}$;

- the INTEGRAL spectrometer (SPI) with an energy resolution of $2.5 \mathrm{keV}$ at $1.3 \mathrm{MeV}$ and an angular resolution of $2.5^{\circ}$ within a field of view of $16^{\circ}$.

Two monitors were included in the payload: JEM-X composed of two identical X-ray telescopes that provide complementary data in the few $\mathrm{keV}$ to $15 \mathrm{keV}$ domain, and OMC, an optical camera for the visible waveband (Winkler et al. 2003).

INTEGRAL was successfully launched from Baikonour (Kazakhstan) on October 17, 2002 using a Proton rocket provided by the Russian Space Agency. The INTEGRAL orbit is eccentric, with an apogee of $153000 \mathrm{~km}$, a perigee of $9000 \mathrm{~km}$, and a 72 hour period (Jensen et al. 2003).

In this paper we first provide a general description of the spectrometer SPI, identifying the main elements of the 
instrument. A second part is devoted to a brief description of each of the main subsystems. Finally we indicate very briefly how SPI is now working in space. A detailed analysis of all the performances of SPI before launch and in space is given by Roques et al. (2003). A detailed presentation of the scientific objectives of SPI can be found in a recent paper by Knödlseder \& Vedrenne (2001).

\section{The spectrometer SPI}

The spectrometer has been realised thanks to a large international collaboration of many institutes that are identified in the next section. A team of the French space agency CNES was responsible for the definition of the mechanical, thermal, and electrical architectures. It followed the development of the subsystems and performed the integration and the tests of the different models. The flight model of SPI was delivered to ESA in May 2001.

Figure 1 gives an overview of the different elements of the spectrometer. The core of the instrument is the gamma camera composed of a hexagonal array of 19 high purity germanium detectors with geometrical area of $508 \mathrm{~cm}^{2}$. As these detectors have to work at low temperature (in the range 85-90 K), an active cryogenic system is used to continuously cool them. Under these conditions the energy resolution for gamma-ray spectroscopy is close to the best which can be achieved today in laboratory, at least 20 times better than the energy resolution of the detectors used in the last two major gamma ray astronomy missions. Even though an active cooling system is technically difficult to operate in space, its redundancy would allow to expect a mission duration of several years.

For imaging, a coded mask made of tungsten blocks is associated with the Ge array. A compromise between the weight and the opacity of the tungsten blocks in the $\mathrm{MeV}$ range led to a thickness of $3 \mathrm{~cm}$ for the blocking elements of the mask.

To increase the sensitivity of the telescope, the background due to cosmic ray interactions with the Ge detectors and all the materials around them has to be reduced. This is achieved with an active anticoincidence system (ACS) made from BGO crystals. The thickness of the BGO crystals was optimised after Monte Carlo simulations of the background (Naya et al. 1996; Jean et al. 1996) because too great a mass of BGO around the detectors can increase secondary neutron production, producing extra background in the Ge crystals. The ACS also defines the field of view of the telescope. As SPI is designed to be able to map extended structures, for instance the ${ }^{26} \mathrm{Al}$ or $511 \mathrm{keV}$ galactic distributions, the field of view has to be large. The chosen fully coded field of view is $16^{\circ}$, with a distance between mask and camera of $1.71 \mathrm{~m}$. This provides an angular resolution of about $2.5^{\circ}$, although sources can be localised much better, to about 10 arcmin depending on the source intensity. Complementary data from IBIS can be used to localise the sources seen by SPI below $200 \mathrm{keV}$ even more precisely $(\sim 1$ arcmin), if the source emits in this energy range.

For background reduction at $511 \mathrm{keV}$, simulations showed the interest of adding a thin plastic scintillator just below the tungsten mask. The Plastic Scintillator Anticoincidence subassembly (PSAC) allows the rejection of background induced

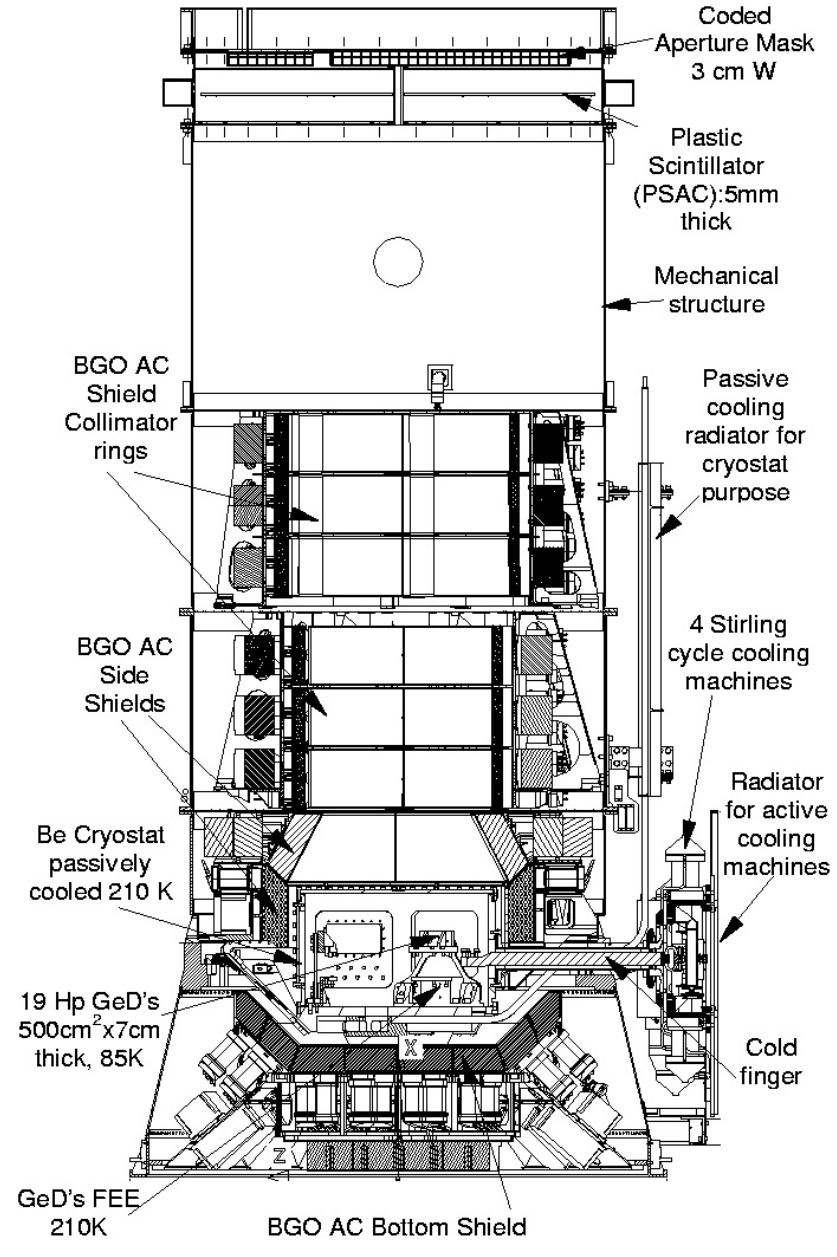

Fig. 1. Cut-out view of the SPI spectrometer.

by prompt interactions in the mask due to particles which do not reach the BGO ACS because of a gap of $70 \mathrm{~cm}$ between mask and ACS (this gap is enforced by weight constraints).

An important source of background which has been identified during the study of gamma-ray detector backgrounds (Gehrels 1985; Gehrels 1992; Naya et al. 1996) are $(n, \beta)$ interactions which dominate the background between $200 \mathrm{keV}$ and $2 \mathrm{MeV}$. If $\beta$ interactions are not accompanied by a detected gamma-ray in the Ge detector they produce a localized energy deposit. They can then be distinguished from interactions of gamma rays which, in this energy range, interact predominantly by Compton scattering followed by a photoelectric interaction of the scattered photon. Such interactions deposit energy in two or more sites. So $\beta$ decays are mostly single site events and most gamma-ray interactions are multiple site events. The shape of the pulses delivered by these two types of events is different and they can be recognised by a pulse shape discrimination (PSD) system. Such a device has been implemented on SPI - this is the first time that this new way to reduce the background between $200 \mathrm{keV}$ and $2 \mathrm{MeV}$ is tried for Ge detectors onboard a satellite.

The operating mode of SPI is based on the detection of Ge detector events which are in the energy range $20 \mathrm{keV}-8 \mathrm{MeV}$ and which are not accompanied by ACS events. The events can be single detector events (SE) with photon energy deposit 


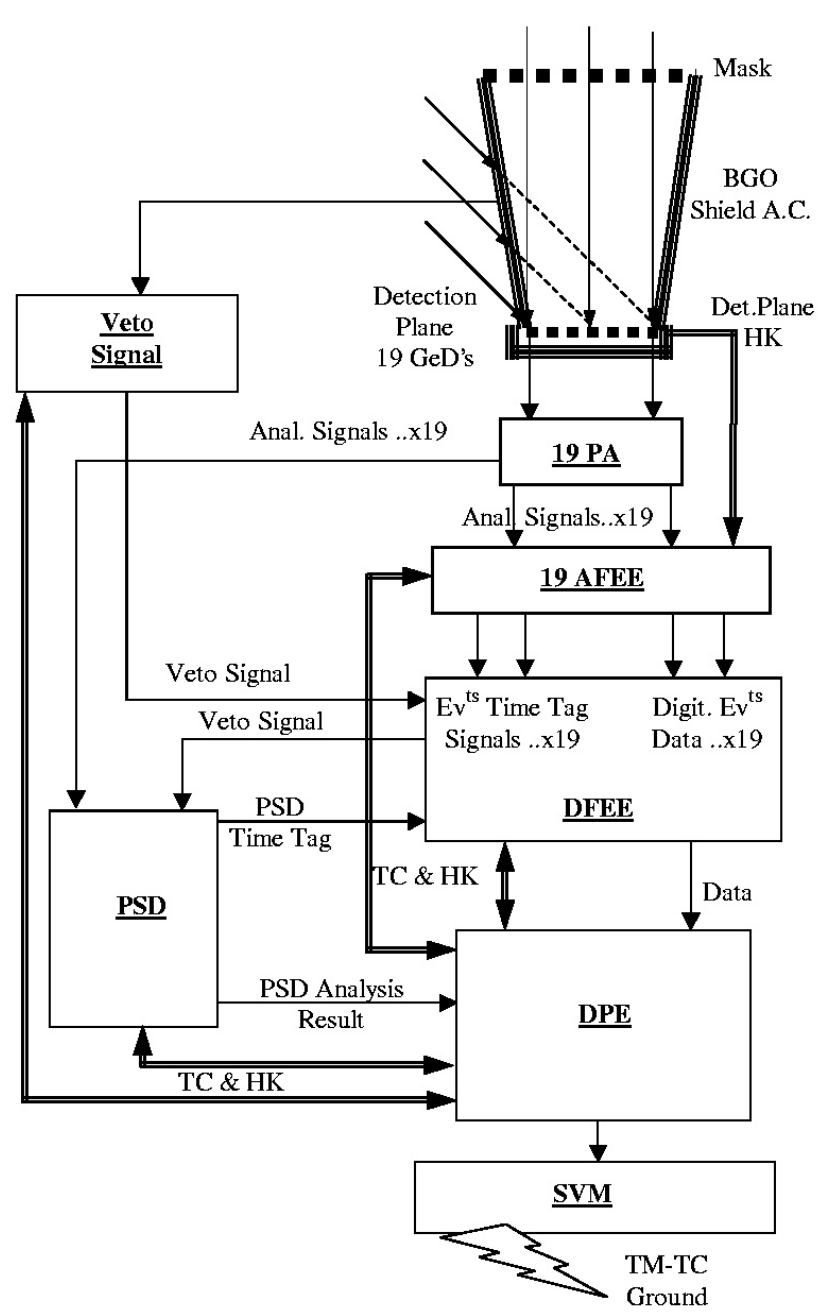

Fig. 2. Block diagram of SPI logics.

in only one detector, or multiple detector events (ME) when a photon interacts through Compton scatterings with energy deposits in more than one detector. In this case, the sum of the energy deposits corresponds to the total energy deposited by the initial photon. Another class of events corresponds to single detector events which are analysed by the PSD (PE) to classify them into single-site interactions (most likely arising from $\beta$ decays) and multiple-site interactions (most likely due to Compton scatterings within a single detector).

All events are processed by the DFEE (Digital Front End Electronics). This system provides event timing with $102.4 \mu \mathrm{s}$ accuracy, event building and classification and event rejection using the ACS veto signal. The events are also counted and the dead time is permanently monitored. The DPE (Data Processing Electronics) receives from the DFEE all the data needed for the onboard analysis prior to telemetry to ground. Figure 2 summarises the functioning principle of the SPI telescope.

The number of detectors in the array is too small to achieve good imaging performance from a single pointing, so successive images of the same field of view are taken with slightly different pointing angles: this is the "dithering" strategy which is usually used for both SPI and IBIS. At intervals of typically $30 \mathrm{~min}$, the spacecraft is reoriented using inertial wheels to
Table 1. Main types of telemetry packets (440 bytes/packet).

Photon packets: all non-vetoed $\mathrm{GeD}$ events (timing accuracy $102.4 \mu \mathrm{s})(80$ packets $/ 8 \mathrm{~s})$

- Single GeD Events (SE): detector address and energy

- Single GeD Events with PSD information (PE)

- Multiple GeD Events (ME): detector addresses and energies within a coincidence time window

Scientific Housekeeping packets: for each second (5 packets/8 s)

- GeD event counters: non-saturating, saturating and non-vetoed

- ACS event counters: non saturating and saturating

- Deadtime: GeD or veto and total

On board spectra from each of the $19 \mathrm{GeDs}$ (non-vetoed or vetoed events) integrated during $\sim 30 \mathrm{~min}$ ( 5 packets $/ 8 \mathrm{~s}$ )

Technological Housekeeping: (3 packets/8 s)

- system status monitoring (temperatures, voltages,...)

- ACS counting rate with $50 \mathrm{~ms}$ time resolution for gamma-ray burst studies

Table 2. SPI instrument characteristics.

\begin{tabular}{ll}
\hline \hline Energy range & $20 \mathrm{keV}-8 \mathrm{MeV}$ \\
Energy resolution & $2.5 \mathrm{keV}$ at $1.33 \mathrm{MeV}$ \\
Angular resolution & $2.5^{\circ}$ \\
Positioning accuracy & $10 \mathrm{arcmin}$ \\
Fully Coded Field-of-view & $16^{\circ}$ \\
Timing & $\pm 52 \mu \mathrm{s}$ \\
Detector area & $500 \mathrm{~cm}^{2}$ \\
Detector thickness & $7 \mathrm{~cm}$ \\
Total weight & $1228 \mathrm{~kg}$ \\
\hline
\end{tabular}

follow a prescribed dithering pattern. This strategy allows the sources to be separated from the background, which is particularly difficult due to the low level of the source signal (at most a few percent of the background). It also minimizes the effects of the small variations of the background which occur despite the choice of an eccentric orbit outside the radiation belts. Finally, for each pointing the spectrum of the events in each detector, ignoring the anticoincidence system, can be recorded.

The different types of telemetry packets provided by the telescope are summarised in Table 1. Table 2 shows the main characteristics of the SPI spectrometer.

After this general description of the spectrometer, in the next section we present each of the main subsystems of SPI in more details.

\section{SPI subsystems}

\subsection{Camera}

The Ge detector (GeD) array consists of 19 reverse-electrode n-type Ge detectors. They have a hexagonal shape with a side length of $3.2 \mathrm{~cm}$ and a height of $69.42 \mathrm{~mm}$. The external diameter is $60.65 \mathrm{~mm}$ and the distance between 2 opposite flat sides 
is $56.04 \mathrm{~mm}$. The dispersion in the detector dimensions is less than $0.5 \%$.

The central bore is $6 \mathrm{~mm}$ in diameter and $5.5 \mathrm{~cm}$ in length. The center to center distance between 2 detectors is $6 \mathrm{~cm}$. Each crystal is individually mounted in an $\mathrm{Al}$ capsule (for technical and cost reasons it was not possible to use Be capsule for the $\mathrm{Ge}$ detectors). The capsules were initially pressurized but due to the leakage of some capsules it was decided to open them to the ambient pressure. This was possible because of the good quality of the Ge crystals and the passivation techniques used. The passivation concerns the rear face of the Ge crystal which must work as a perfect insulator. Pollution of this part produces leakage currents which can greatly affect the energy resolution.

The hexagonal shape was chosen to minimize the volume occupied by the Ge array and therefore the volume and the weight of the anticoincidence system which surrounds the camera. The closely packed array of Ge detectors is fixed on a Be plate and the detector plane is put inside a cryostat which is also made of $\mathrm{Be}$ to minimize the background. The plate is connected to the Stirling cycle cryocoolers through a Be cold finger electrically insulated by a sapphire interface.

The ultra-pure germanium was supplied by Hoboken - Union Miniere in Belgium, under the responsibility of P. Leleux (UCL, Belgium). The impurity level was specified to be between $5 \times 10^{9} \mathrm{~cm}^{-3}$ and $1.5 \times 10^{10} \mathrm{~cm}^{-3}$. This impurity level and the small diameter of the central bore were specified in order to optimize the operation of the PSD. The mean volume of the detectors is $178 \mathrm{~cm}^{3}$ and the mean crystal weight is $951 \mathrm{~g}$, with a dispersion of $2.6 \%$. The total weight of $\mathrm{Ge}$ is $18 \mathrm{~kg}$. The mean weight of each housed detector is $1133.6 \mathrm{~g}$ with a dispersion less than $2.9 \%$. The total geometrical area for a flux parallel to the axis of the Ge array is $508 \mathrm{~cm}^{2}$. The Ge detectors were constructed by Eurysis Mesures in France.

To collect efficiently the electrons and holes which are produced following the interaction of a photon or charged particle in the crystal, it has to be fully depleted of free charges. This requires a sufficient voltage applied between the inner and outer electrodes. The Ge detectors of SPI normally work at $4000 \mathrm{~V}$. This high voltage is variable between zero and $5000 \mathrm{~V}$.

The energy resolution of GeD's decreases when the detectors are damaged by radiation. To minimise this effect, which is due mainly to hole traps, the length of Ge traversed by the holes has to be minimized to reduce the probability of trapping. As most of the volume of a hexagonal or cylindrical detector is near the outer radius, most interactions take place near the outside. n-type Ge detectors were chosen for SPI so that holes move towards the external electrode and thus typically have to travel a short distance before collection.

Although minimizing the path length for the holes reduces the effects of radiation damage, it is not enough to preserve the performance over long times (a year or more). The only way to avoid cumulative effects of radiation damage is to anneal the detectors. This means holding them at $105^{\circ} \mathrm{C}$ during one or 2 days, depending on the level of damage. We have measured that after damage leading to a $20 \%$ increase in the FWHM of a line at $1.3 \mathrm{MeV}$ an annealing with a duration of 2 days is enough to fully recover the energy resolution of the detector
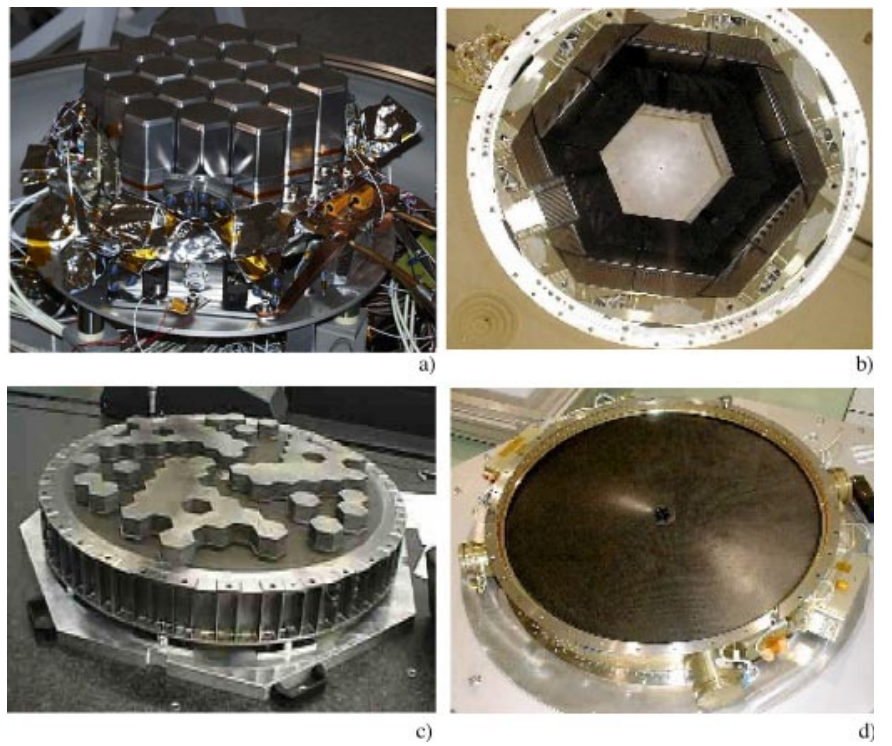

Fig. 3. View of some subsystems of the spectrometer: a) Ge array, b) ACS, c) mask, d) plastic scintillator.

(Leleux et al. 2003). This annealing technique has been implemented for SPI and successfully tested.

The Ge array is mounted on a Be plate (Fig. 3a) which on the bottom side houses the first stage of the preamplifier circuitry (PA1), which has only passive components (high voltage filter, charge resistor and coupling capacitor mounted on a printed circuit board). The second stage of the preamplifiers (PA2) is on a second plate stabilised at $210 \mathrm{~K}$ and thermally connected to the cryostat. This stage includes the FET, for which the low temperature allows a reduction of the electronic noise. The PA2 delivers:

- the energy signal which will be further amplified; at this point the typical signal height is $50 \mathrm{mV}$ for a $1 \mathrm{MeV}$ energy deposit in a Ge detector;

- the fast signal which is delivered to the PSD.

To avoid strong saturation of the preamplifiers due to large energy deposits in the Ge crystal by charged particles a desaturating system is implemented. It performs a reset for pulses corresponding to more than $14 \mathrm{MeV}$ energy deposit in the $\mathrm{Ge}$ crystal. The count rate of saturating events can be used to follow in orbit the evolution of the Ge background.

\subsection{Analogue Front End Electronics (AFEE)}

The AFEE receives the 19 signals from the PA2. The goal of the 19 electronic chains of the AFEE is the amplification and the filtering of the signals. This is done in the Pulse Shape Amplifiers (PSA). The pulse shaping is Gaussian with a peaking time of $8 \mu \mathrm{s}$. The PSA also provides a timing signal used for the time-tagging of each analysed event. The pulse heights corresponding to energies between 0 and $8 \mathrm{MeV}$ are coded by the Pulse Height Analyser (PHA) with 14 bits (16384 channels in two energy ranges: $0-2 \mathrm{MeV}$ and 2-8 MeV). For the first energy range each channel has a resolution of $0.13 \mathrm{keV}$, while for the second range one channel corresponds to $0.52 \mathrm{keV}$. 
A photon below $8 \mathrm{MeV}$ is processed in $26 \mu \mathrm{s}$. In order to ensure a good recovery of the analogue amplifier after a saturating event (event energy in excess of $8 \mathrm{MeV}$ ), the electronics is blocked during $100 \mu$ s for each such event. The AFEE also supplies low voltage power for the preamplifiers and high voltage for the Ge detectors.

The camera, including the detection plane and its electronics (Preamplifiers and AFEE) was under the responsibility of J. P. Roques at CESR in Toulouse (France).

\subsection{Pulse Shape Discrimination electronics (PSD)}

SPI is equipped with a Pulse Shape Discrimination (PSD) electronics that aims at a reduction of the instrumental background in the energy range 200-2000 keV. Simulations of the instrumental background indicate that in this energy range the dominant background component consists of localised beta decays, i.e. beta decays that are not accompanied by emission of a gamma-ray photon. In this case, the energy deposit within the germanium detector is localised within a few mm, leading to a single current pulse in the pre-amplifier. Gamma-ray photons, in contrast, interact primarily by Compton scatterings in this energy range, leading to multiple interactions within the detector volume. The current pulses for each of the interactions superimpose, leading to a broadening of the observed pulse shape. For each of the events in the 200-2000 keV domain, PSD is measuring the current pulse shape and subjecting it to an analysis in order to determine if a localised or a non-localised interaction occurred in the detector. During data analysis on ground, this information may then be used to enhance the sensitivity of the telescope.

The PSD electronics interfaces directly with the fast output of the pre-amplifiers that provide an image of the current pulses occurring in the Ge detectors. The 19 input signals feed into a triggering and multiplexing electronics that activates pulse shape analysis if a current pulse has been detected on one and only one of the input channels (hence multiple detector triggers are not analysed by the PSD electronics). In case of a trigger, the input current is digitised for a period of about $1 \mu \mathrm{s}$ with a 10 ns time resolution. This covers largely the typical pulse duration of about $300 \mathrm{~ns}$ and provides sufficient data points for determination of the baseline current. The digitised pulses are stored in a buffer where they await their asynchronous analysis using a Digital Signal Processing unit (DSP) clocked at a frequency of $40 \mathrm{MHz}$.

Upon event trigger, the PSD electronics sends a time-tag and an event identification label to the DFEE electronics that will be used later to combine the PSD information with that coming from the AFEE electronics. After pulse shape analysis, the PSD electronics transmits the analysis results to the DPE, where they will be combined by means of the event identification labels with the information provided by the DFEE electronics. Thus, the final building of the PSD events (PE) is performed in the DPE. This logic implies that the PSD electronics does not add any deadtime to the detection chain. In the case that an event was lost by the PSD electronics (because it occurred for example during the $\sim 80 \mu$ s digitisation time during which the PSD electronics cannot accept a new event), no PSD information will be provided to the DFEE and DPE, and the corresponding event will be classified as single event (SE). Thus, a certain fraction of the events in the energy range 200-2000 keV will contain no PSD information.

The pulse shape analysis and discrimination is performed by a DSP using a patchable assembler code. Each pulse shape is baseline subtracted and time aligned and then compared to pairs of so-called template pulse shapes that are stored in a patchable on-board library, burned into EEPROM. The template pulses consist of average pulse shapes for localised interactions, classified by the pulse rise time. The pulse rise time is a crude measure of the radial distance of the interaction site to the central bore of the detector. Typically, 25 templates are sufficient to cover all possible pulse shapes that may occur in the detector. To accommodate for detector-to-detector variations, each of the 19 detection channels has a dedicated template library. The analysis algorithm determines the best fitting pair of templates based on a $\chi^{2}$ optimisation scheme. As analysis results, the best fitting template indices and their relative amplitudes are encoded in a 16 Bit word and transmitted to ground. The analysis code has been highly optimised for speed and performs pulse shape analysis within less than $1 \mathrm{~ms}$.

Initial template libraries have been built during ground calibration measurements at ESTEC (Attié et al. 2003). Inflight, a special PSD calibration mode has been foreseen that allows downlinking of digitised pulse shapes for template building. No experience exists so far on the variation of the pulse shapes with time, yet it is expected to perform a PSD calibration after each annealing cycle.

The design and fabrication of the PSD were under responsibility of B. Lin (University of California Berkeley, USA) and J. Matteson (University of California San Diego, USA), respectively, while integration and testing were supervised by J. Knödlseder at CESR in Toulouse (France).

\subsection{Cryostat}

The cryostat, under CNES responsibility (Briet 1999), was designed to allow the Ge array to be cooled to temperatures below $100 \mathrm{~K}$, if possible reaching $85 \mathrm{~K}$. The latter temperature is desirable to slow the effects of radiation damage and to increase the time between successive annealings in space. The cooling is obtained thanks to 4 mechanical Stirling cycle coolers delivered by MMS - Astrium under ESA responsibility. The cryostat also provides the functions necessary for Ge crystal annealing.

The four coolers are mounted on an external radiator (ACC) and are operated in pairs (SPICO 1 and 2) to reduce microphonic noise on the detectors. Each SPICO shares a common Cooler Drive Electronics (CDE) located on the Payload Module (PLM).

The performance of the mechanical coolers in terms of heat lift is limited to a few Watts, so the Ge detectors have to be very well thermally insulated from their environment. In order to minimise the heat leaks, the detection plane is enclosed in 
a beryllium structure (Cold Box) controlled at an intermediate temperature $(210 \mathrm{~K})$ through a passive cooling device (PAC).

A lot of care was necessary to minimize the thermal leakage between the detector plane and the Cold Box. Multilayer isolation is used around the cold stage. The mechanical supports are in fibreglass and between PA1 and PA2 Manganin wires with small cross sections are used. Thermal connection between the Cold Box and the external radiator is realised by two ammonia-filled heat pipes. They carry the heat due to the preamplifiers PA2 and the thermal leakage which occurs between the Cold Box and the main structure of SPI despite multilayer insulation (MLI) and the low thermal conductivity of the Ti supports of the Cold Box. The heat load on the cryocoolers, and thus the power consumption, can be significantly reduced by keeping this intermediate stage as cold as possible. Consequently the heat pipes are used near to the ammonia freezing point $(196 \mathrm{~K})$. This requires an antifreeze safety system with electronic regulation which is used in all observational phases. Nevertheless, freezing of the ammonia can occur when not in operating modes. Thus a heat pipe thaw system was necessary to allow the heat pipes to be brought into operation after the coast phase or after entry into survival mode.

The regulation of the annealing to recover detector performance after radiation damage is done thanks to an electronic circuit located in the AFEE. Moreover the use of unsealed Ge detectors means that adequate outgassing of the detectors and of the cryostat was essential before the start of operations. The cold stage outgassing is achieved by the same heaters that are used during the annealing process.

To summarise, the SPI concept led to a design with 3 stages (Fig. 4): (i) a cold stage at $85-90 \mathrm{~K}$ (GeD plane and cold link to coolers, the cold finger, which has been called Cold Rod) (ii) an intermediate stage at $210 \mathrm{~K}$ (Cold Box, heat pipes and passive radiator) and (iii) an ambient temperature stage at $300 \mathrm{~K}$ (the coolers and their associated structures and the radiator). The mass of the cryostat without detectors is $80 \mathrm{~kg}$. The 19 detectors add a further $22 \mathrm{~kg}$.

The cryostat was under CNES responsibility (R. Briet and D. Arrat).

\subsection{Anticoincidence shield}

The large hexagonal anticoincidence shield (ACS) consists of 91 BGO crystals each with a volume of $\sim 790 \mathrm{~cm}^{3}$ which are arranged in 4 subunits (Fig. 3b). The units of the upper veto shield (UVS) consist of the upper collimator ring (UCR), the lower collimator ring (LCR) and the side shield assembly (SSA), each containing 18 crystals which are arranged hexagonally around the cylindrical axis of SPI. The lower veto shield (LVS), consisting of 36 crystals, is assembled as a hexagonal shell. The thickness of the crystals increases from $16 \mathrm{~mm}$ at the top (UCR) to $50 \mathrm{~mm}$ at the bottom (LVS). The total mass of BGO used for the ACS is $512 \mathrm{~kg}$.

Each BGO crystal of the ACS (with one exception) is viewed by two photomultipliers (PMTs). Due to the redundancy concept used for the ACS, each of the 91 front-end electronic boxes (FEEs) sums the anode signals of two PMTs,

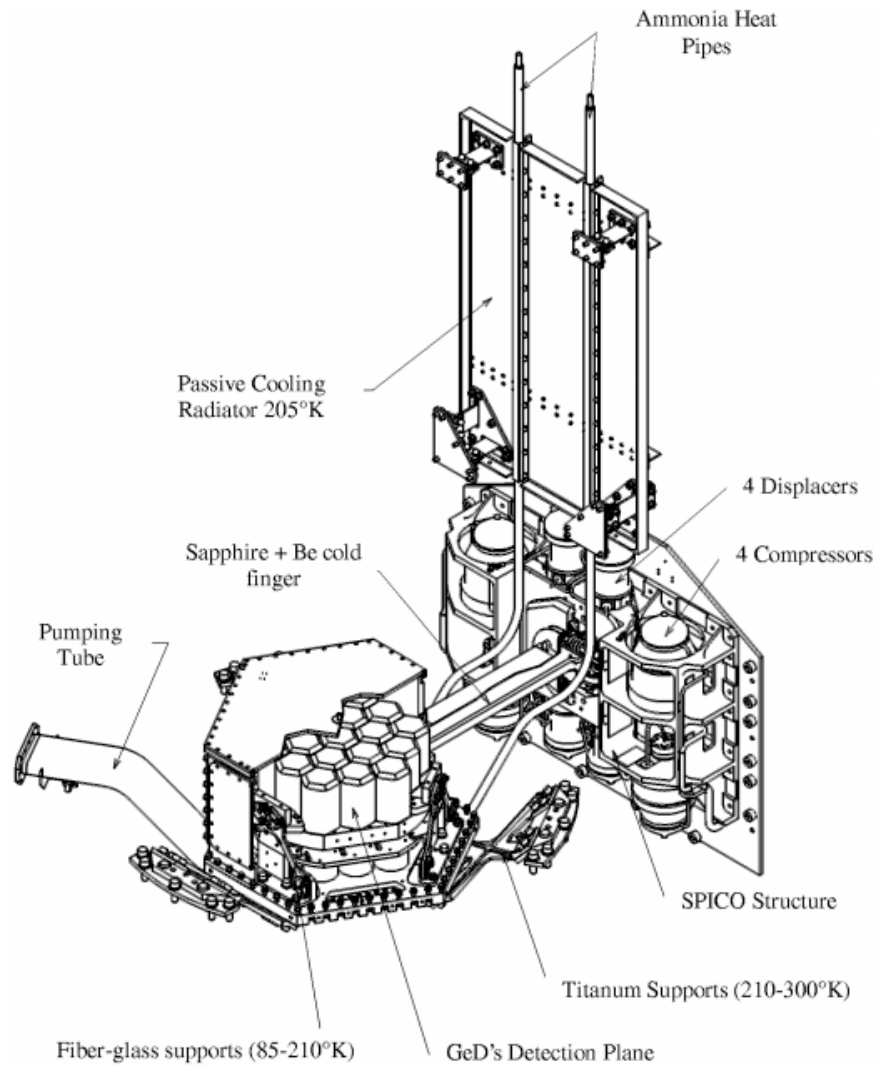

Fig. 4. The SPI cooling system.

which are viewing different BGO crystals (in most cases neighbouring crystals). In case of a failure of one single PMT or FEE, this cross strapping of FEE and BGO units means that no BGO crystal would be lost. It emerges that a disadvantage of this method is an uncertainty in the energy-threshold value of individual FEEs, caused by a different light yield of neighbouring BGO-crystals and different PMT properties like quantum efficiency and amplification. A result of this is that the threshold extends over an energy range of the order of $100 \mathrm{keV}$ and is not at all sharp. The energy-threshold settings of the ACS depend on a tradeoff between background reduction and deadtime for the SPI camera. An optimal adjustment was achieved during the commissioning phase with a threshold setting of about $75 \mathrm{keV}$.

One important contribution of the ACS to the science obtained with SPI is its use as a gamma-ray burst monitor. The ACS provides a large effective area for the detection of bursts (von Kienlin et al. 2003; von Kienlin et al. 2001; Hurley 1997; Lichti et al. 2000), but unfortunately with no (or only very coarse) positional information. But by using the 3rd interplanetary network, to which the SPI ACS has been added, one can derive burst locations down to the arcmin range.

The ACS was developed at MPE (Garching, Germany) under the responsibility of G. G. Lichti and A. von Kienlin. 


\subsection{Mask}

The SPI mask, which is situated at $1.71 \mathrm{~m}$ above the detection plane is a circular passive array of 127 hexagonal pixels of which 63 are opaque and 64 transparent to gamma radiation within the operational energy range (Fig. 3c). The HURA (Hexagonal Uniform Redundant Array ) coded mask pattern has $120^{\circ}$ symmetry and is inscribed in a $720 \mathrm{~mm}$ diameter circle. The cells are $60 \mathrm{~mm}$ side to side, the same as the detector module pitch. The opaque cells are made of a tungsten alloy (density $18 \mathrm{~g} \mathrm{~cm}^{-3}$ ) and are $30 \mathrm{~mm}$ thick. This allows an opacity better than $90 \%$ over the entire energy range. The tungsten cells are mounted on a carbon fibre sandwich assembly. The measured positioning accuracy (mean deviation over the theoretical position) of such tungsten cells was $0.04 \mathrm{~mm}$ (the required value during the design phase was $0.15 \mathrm{~mm}$ ).

To align the detection axis of SPI it was decided to have a central hole in the mask to enable a fiducial mark on the cryostat to be sighted through the mask and the PSAC which is just below the mask. This hole (diameter $10 \mathrm{~mm}$ ) is reinforced with potting (external diameter $28 \mathrm{~mm}$ ) and titanium washers (diameter, $40 \mathrm{~mm}$ ) on both lower and upper parts of the sandwich panel. This structure decreases the transparency of the central element of the mask with respect to the rest of the open mask elements (from $82 \%$ to $59 \%$ at $60 \mathrm{keV}$ and from $88 \%$ to $79 \%$ at $511 \mathrm{keV})$. Except for this central pixel the transparency of the mask is in agreement with the specifications. For instance at $0^{\circ}$ the transparency is $75 \%$ at $35 \mathrm{keV}$, reaching $85 \%$ at $300 \mathrm{keV}$ (Sanchez et al. 2000). The mask weight is about $140 \mathrm{~kg}$.

The mask was developed under the responsibility of F. Sanchez, University of Valencia (Spain).

\subsection{Plastic scintillator}

Monte Carlo simulations have shown that a significant background reduction can be achieved, especially at $511 \mathrm{keV}$ (Jean et al. 1997), if a plastic scintillator is placed just below the mask. This circular plastic scintillator, $5 \mathrm{~mm}$ thick and $800 \mathrm{~mm}$ in diameter, is situated inside a light diffusion chamber (106 $\mathrm{mm}$ height and $826 \mathrm{~mm}$ in diameter) with a millipore paper coating (Fig. 3d). Four photomultipliers with $70 \mathrm{~mm}$ diameter photocathodes are distributed around the diffusion box, not in contact with the plastic scintillator. The diffusion chamber assures a uniform distribution of photons produced by particle interactions in the thin plastic scintillator and minimizes the dispersion in the response for interactions at different positions in the scintillator. Here again a central hole (diameter $12 \mathrm{~mm}$ ) allowed the alignment of SPI. The absorption due to the material around this hole (diameter $26 \mathrm{~mm}$ ) has been measured and it is comparable to the absorption of the central hole of the mask. To have redundancy for this subsystem also, it was decided after many tests to use only 2 photomultipliers in opposite directions to build the veto signal and to keep the 2 others for redundancy. This was possible because the mean energy deposit of charged particles is large enough $(1 \mathrm{MeV})$ to be above the electronic threshold of the PSAC electronics $(300 \mathrm{keV})$ independent of the position of the particle interaction in the plastic. The PSAC weight is $22 \mathrm{~kg}$. This subsystem was

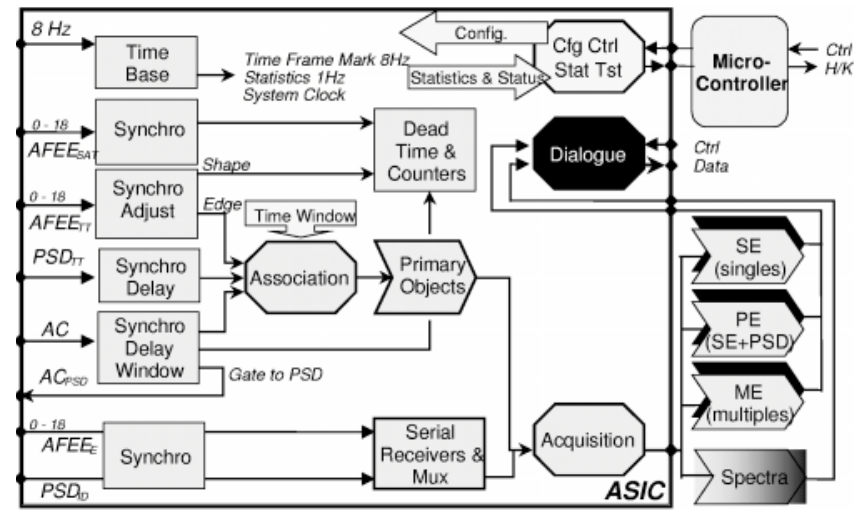

Fig. 5. Block diagram of the SPI DFEE.

realised under the responsibility of P. Caraveo, IASF (Milano, Italy).

\subsection{Digital Front End Electronics (DFEE)}

The block diagram of the SPI-DFEE (Schanne et al. 2002) with its digital ASIC is shown in Fig. 5. The front stage of the DFEE receives the ACS veto signal (dead time of $725 \mathrm{~ns}$ per hit), the 19 time tags sent by the AFEE and a time tag sent by the PSD for each analysed event. Each signal is delayed internally by a time value adjustable in steps of $50 \mathrm{~ns}$.

The output signals of the DFEE front stage are used by the "association machine" of the DFEE in order to perform the event building. A time tag is considered to correspond to a Single Detector Event (SE) if no other time tag occurs within the duration of an adjustable "association time window" (typically $350 \mathrm{~ns}$ ). The event is a Multiple Detector Event (ME) if a subsequent time tag arrives before the closure of the association time window opened by a previous time tag. A SE with a PSD-Time Tag arriving during the association time window is classed as a Single Detector Event with PSD information (PE). The absolute time of an event classified as SE, ME or PE is measured by the DFEE in units of $102.4 \mu$ s with respect to an onboard $8 \mathrm{~Hz}$ clock. Within a ME the time between subsequent time tags is measured in units of $50 \mathrm{~ns}$. All events are stored into an internal FIFO in order to wait for the arrival of the numerical values of the energies determined by the AFEE, afterwards they are extracted from the FIFO and written into output tables together with their energy values. Those tables are handled in a dead-time free double-buffer mode and sent on the basis of a time frame defined by the onboard $8 \mathrm{~Hz}$ clock to the Digital Processing Electronics (DPE). The DPE assembles the PE information with the result of the PSD analysis and sends the resulting stream to the satellite telemetry downlink system.

The high speed data processing of the DFEE is entirely handled by a digital ASIC (Lafond et al. 1998; Mur et al. 2002) designed at CEA Saclay and built by TEMIC/Atmel in a radiation tolerant CMOS technology compatible with the space environment (maximal integrated dose $0.3 \mathrm{~Gy}$ ). The DFEE was realised under the responsibility of CEA, France (B. Cordier and S. Schanne). 


\section{SPI in space}

As a conclusion we can add a few words on the behaviour of SPI after the launch in October 2002. After an outgassing period of 15 days, the coolers were successfully activated. When the temperature of the detectors array reached $115 \mathrm{~K}$, high voltages were switched on, starting progressively from zero to 4000 Volt. All the detectors worked well except detector number 15, which had already shown the largest susceptibility to pollution during laboratory tests. To optimise its energy resolution, a lower high voltage of 2500 Volt has been chosen. This clearly demonstrated that the outgassing phase was not long enough to clean completely all the 19 detectors. After 3.5 months it was decided to perform an annealing. It started on the 6th February and lasted 11 days, with an annealing period of 37 hours. All the detectors recovered completely their performance; in particular the pollution of detector 15 disappeared and its high voltage was also set to 4000 Volt.

All subsystems work nominally. The performance of the ACS subsystem after launch was as expected and it is still properly functioning, showing no technical weakness or a part failure in the first 6 months of the mission. The background of SPI's camera is reduced by the veto shield by a factor of about 25 (Jean et al. 2003).

The instrumental background turned out to exceed the most recent simulations (Weidenspointner et al. 2003) by 30-40\%. In particular, the background associated with the main lines produced by neutron interactions with the Ge crystals is considerably larger than anticipated. This discrepancy is explained by a large contribution from neutron reactions.

Background reduction by the PSD sub-assembly seems to be modest if not negligible, which can be explained by a relatively low fraction of localised $\beta$-decays in the PSD energy range with respect to model predictions. Also the PSAC, which has as primary aim the reduction of the instrumental $511 \mathrm{keV}$ background line, proved only of modest efficiency. Nevertheless, the observed level of the $511 \mathrm{keV}$ background line is closed to the predicted value. The sensitivity of SPI for narrow lines and continuum is presented in the accompanying paper on the SPI inflight performances (Roques et al. 2003).

The increased background (with respect to pre launch simulations) leads to a bottleneck in the data transmission due to the limited telemetry bandwidth. Consequently, some efforts have been made to use the available telemetry more efficiently and to gain access to more packets in order to allow the transmission of SPI data in the photon by photon mode (this mode is essential to get the maximum performance from the instrument).
In spite of this background increase, the spectrometer is functioning extremely well.

Acknowledgements. The SPI project has been completed under the responsibility and leadership of CNES. We are grateful to ASI, CEA, CNES, DLR, ESA, INTA, NASA and OSTC for support.

\section{References}

Attié, D., Cordier, B., Gros, M., et al. 2003, A\&A, 411, L71

Briet, R. 1999, in Cryogenic Engineering Conf. 1999, Montreal

Gehrels, N. 1985, NIM A, 239, 324

Gehrels, N. 1992, NIM A, 313, 513

Hurley, K. 1997, in Proc. 2nd INTEGRAL Workshop, The Transparent Universe, St. Malo, France, 16-20 September 1996, ed. C. Winkler, T. Courvoisier, \& Ph. Durouchoux, ESA SP 382, 491

Jean, P., Naya, J., von Ballmoos, P., et al. 1996, SPIE, 2806, 457

Jean, P., Naya, J., von Ballmoos, P., et al. in Proc. 2nd INTEGRAL Workshop, The Transparent Universe, St. Malo, France, 16-20 September 1996, ed. C. Winkler, T. Courvoisier, $\&$ Ph. Durouchoux, ESA SP 382, 635

Jean, P., Vedrenne, G., Roques, J. P., et al. 2003, A\&A, 411, L107

Jensen, P. L., Clausen, K., Cassi, C., et al. 2003, A\&A, 411, L7

Knödlseder, J., \& Vedrenne, G. 2001, in Proc. of the 4th INTEGRAL Workshop, Alicante (Spain), ESA SP 459, 23

Lafond, E., Mur, M., \& Schanne, S., IEEE Transact. Nucl. Sci., vol. $45,4,1836$

Lichti, G. G., Georgii, R., von Kienlin, A., et al. 2000, in Proc. 5th Compton Symp., Portsmouth, NH ed. M. L. McConnell, \& J. M. Ryan, AIP Conf. Proc., 510, 722

Mur, M., Cordier, B., Donati, M., et al. 2002, IEEE Transact. Nucl. Sci., 49(5), 2492

Naya, J., Jean, P., Buckolt, J., et al., NIM A, 368, 832

Roques, J. P., Schanne, S., von Kienlin, A., et al. 2003, A\&A, 411, L91

Sanchez, F., Chato, R., \& Gasent, J. L. 2000, SPI Mask STM/QM Transparency Test Report, IN-SP-UV-RPT-049

Schanne, S., Cordier, B., Gros, M., et al. 2002, IEEE Transact. Nucl. Sci., 49(3), 1226

von Kienlin, A., Arend, N., Lichti, G. G., Strong, A., \& Connell, P. 2003, SPIE Conf. Proc., 4851

von Kienlin, A., Arend, N., \& Lichti, G. G. 2001, in Gamma-Ray Bursts in the Afterglow Era, Proc. of the International workshop held in Rome, CNR headquaters, 17-20 October, 2000, ed. E. Costa, F. Frontera, \& J. Hjorth (Berlin Heidelberg: Springer), 427

Weidenspointner, G., Kiener, J., Gros, M., et al. 2003, A\&A, 411, L113

Winkler, C., Courvoisier, T. J.-L., Di Cocco, G., et al. 2003, A\&A, 411, L1 